\title{
Karsten Weber
}

\section{Entwicklung und digitale Spaltung - Zusammenhänge und Prioritäten}

\section{Situationsbeschreibung}

Im Internet findet man alles - das weiß inzwischen jeder Mensch. Deshalb ist es nicht überraschend, dass es im Internet unter der URL http://www.wsis.org eine Website gibt, auf der offiziell der World Summit on the Information Society (WSIS) dokumentiert und auf weitere Aktivitäten verwiesen wird, Ergebnisse dargestellt und viele andere Informationsangebote gemacht werden. Es wäre aber kaum der Mühe wert, eine Umfrage durchzuführen, wer von den ganz normalen Menschen auf der Straße etwas über den WSIS und seine Ziele wüsste. Das Ergebnis ist auch so leicht vorauszusagen: Sehr wenige bis niemand. Dabei waren die Ziele des WSIS ambitioniert - es sollten Mittel und Wege aufgewiesen werden, die helfen könnten, die digitale Spaltung der Welt in 'information haves' und 'information have-nots' bzw. in 'information rich' und 'information poor' aufzuheben oder zumindest den tiefen Graben zwischen diesen beiden Gruppen kleiner zu machen.

Es muss jedoch nicht sonderlich verwundern, dass der WSIS in der Öffentlichkeit vergleichsweise wenig Beachtung gefunden hat und ein Thema geblieben ist, das allenfalls Experten und Betroffene interessiert. Zumindest in Deutschland kann man eine ähnliche Nichtbeachtung in Bezug auf Bildung beobachten: Für eine kurze Zeit wurde in Deutschland nach dem so genannten PISA-Schock über Bildungsdefizite debattiert, doch inzwischen ist diese Debatte entweder ganz untergegangen oder wird nur mehr rein instrumentell geführt - Bildung wird als Ressource angesehen, die ein Land für die eigene ökonomische Leistungsfähigkeit benötigt. Ähnliches ist im Zuge der kurz aufflammenden weltweiten Diskussion um die digitale Spaltung zu beobachten gewesen. Von ihrer Schließung wurde die Lösung so unterschiedlicher gesellschaftlicher Probleme wie Arbeitslosigkeit, fehlendes wirtschaftliches Wachstum (Zachary 2004), Defizite des Bildungswesens, mangelnde Berufsqualifikation (Darkwa/Eskow 2000; Darkwa/Mazibuko 2000), Politikverdrossenheit oder fehlendes Bürgerengagement angesehen (kritisch: Gurstein 2003); wahrscheinlich ließe sich die Liste auch noch deutlich verlängern. Ebenso wurde immer wieder die Hoffnung geäußert, dass die Behebung der digitalen Spal- 
tung helfen werde, den Analphabetismus global zu bekämpfen, die weit verbreitete Armut zu beheben, die schwächelnde Weltwirtschaft anzukurbeln, zur weltweiten Demokratisierung beizutragen und endlich den Menschenrechten stärker zur Geltung zu verhelfen - auch hier ist die Agenda der Hoffnungen wohl beliebig erweiterbar. Es ist kaum übertrieben zu sagen, dass von der Schließung der digitalen Spaltung die Lösung beinahe jedes ökonomischen, rechtlichen, politischen, sozialen oder moralischen Konflikts oder Problems erwartet wurde. Anders formuliert: Der Einsatz einer bestimmten Technologie, hier der Informations- und Kommunikationstechnologie, sollte tief greifende soziale Konflikte lösen. Diese Sichtweise manifestiert sich bspw. in einer Stellungnahme des US Department of Commerce in Bezug auf die USamerikanische Gesellschaft:

„The Internet is becoming an increasingly vital tool in our information society. More Americans are going online to conduct such day-to-day activities as business transactions, personal correspondence, research and information-gathering, and shopping. Each year, being digitally connected becomes ever more critical to economic, educational, and social advancement. Now that a large number of Americans regularly use the Internet to conduct daily activities, people who lack access to those tools are at a growing disadvantage. Therefore, raising the level of digital inclusion - by increasing the number of Americans using the technology tools of the digital age is a vitally important national goal." (USDC 2000: 14)

Ähnliche Ansichten finden sich bspw. in einem Bericht des Research Institute for Asia and the Pacific (RIAP 2002) der Universität Sydney, der vom japanischen Finanzministerium in Auftrag gegeben wurde und die Bedeutung der digitalen Spaltung für die ökonomische Situation Malaysias, Thailands, der Philippinnen und Vietnams behandelt sowie mögliche Entwicklungspfade diskutiert. Nun ist eine solche Sichtweise für ein Handels-, Wirtschafts- oder Finanzministerium kaum überraschend, doch die gleiche Perspektive findet sich in der „Declaration of Principles - Building the Information Society: a global challenge in the new Millennium“, die als eines der wenigen wenigstens einigermaßen konkreten Ergebnisse des World Summit on the Information Society angesehen werden kann:

„2. Our challenge is to harness the potential of information and communication technology to promote the development goals of the Millennium Declaration, namely the eradication of extreme poverty and hunger; achievement of universal primary education; promotion of gender equality and empowerment of women; reduction of child mortality; improvement of maternal health; to combat HIV/AIDS, malaria and other diseases; ensuring environmental sustainability; and development of global partnerships for development for the attainment of a more peaceful, just and prosperous world." (WSIS 2003)

Nun könnte man formulieren, dass entsprechende Sätze einer allgemeinen und dabei auch recht unspezifischen Hoffnung auf Verbesserung in den Entwicklungsländern geschuldet seien. Doch selbst bei Autoren ${ }^{1}$, die eine eher kritische Haltung gegenüber der gerade skizzierten Sichtweise an den Tag le-

1 In diesem Text wird aus Gründen der Lesbarkeit einheitlich die maskuline Form genutzt. Gemeint sind jedoch immer alle Geschlechter. 
gen, finden sich Ansätze, diese Perspektive zur eigenen zu machen:

„[t]he differentiation between Internet-haves and have-nots adds a fundamental cleavage to existing sources of inequality and social exclusion in a complex interaction that appears to increase the gap between the promise of the Information Age and its bleak reality for many people around the world." (Castells 2001: 247)

Auch für Castells ist das Informationszeitalter, als Chiffre für die Entwicklung und den Einsatz einer bestimmten Technologie, mit einer bestimmten Hoffnung verbunden - nämlich der Verbesserung des Lebens für all jene Menschen, die am unteren Ende der sozialen Skala leben. Zwar verbindet Castells diese Hoffnung nicht nur mit der Technologie, sondern eher mit den dadurch möglichen neuen Austauschprozessen zwischen Menschen; trotzdem zeigt seine Perspektive Ähnlichkeiten zu jenen auf, die oben stellvertretend angeführt wurden. Sowohl die Position des USDC als auch jene des WSIS basiert letztlich auf einem Technikdeterminismus mit positivem Vorzeichen: Es wird angenommen, dass die Entwicklung, Implementierung und Nutzung von Technologie notwendig zur Lösung der sozialen Probleme einer Gesellschaft oder gar der Menschheit führen werden und die Basis sozialer und politischer Veränderungen darstellen (vgl. Carlisle/Manning 1999: 100). Dies knüpft an Ideen an, die man etwa mit dem Namen Saint-Simon verbinden kann, die dann im Marxismus ihren prägnanten Ausdruck fanden und bis heute im Fundus der Soziologie und Philosophie zu finden sind. So waren die 1960er und 70er Jahre geprägt durch die Idee, dass der massive Einsatz von Technologie und die vollständige Planung aller gesellschaftlichen Lebensbereiche alle wesentlichen sozialen Phänomene (auf)lösen können. Dies muss umso mehr verwundern, da bereits in dieser Zeit mit Publikationen wie Rachel Carsons Silent Spring (1962) oder der Studie The Limits to Growth (Meadows et al. 1972; deutsch: Die Grenzen des Wachstums) des Club of Rome aufgezeigt wurde, dass ein bloßes Mehr an Technik keine Probleme lösen kann, sondern eher neue entstehen lässt.

Wie wenig haltbar die optimistischen Annahmen sind, die in der öffentlichen Debatte - bspw. auf dem G8-Gipfel auf Okinawa im Jahr 2000 - häufig eingenommen werden und wie vereinfachend die Perspektiven auf komplexe Problembereiche aussehen, zeigt sich bei einer kritischen Betrachtung der Ursachen und Auswirkungen der digitalen Spaltung. Gleich auf den ersten Seiten seines Buch Technology and Social Inclusion schreibt Mark Warschauer, dass

„[...], the notion of a digital divide - even in its broadest sense - implies a chain of causality: that lack of access (however defined) to computers and the Internet harms life chances. While this point is undoubtedly true, the reverse is equally true; those who are already marginalized will have fewer opportunities to access and use computers and the Internet. In fact, technology and society are intertwined and co-constitutive, and this complex interrelationship makes any assumption of causality problematic." (Warschauer 2003: 7)

Ökonomische und informationelle, oft auch politische und kulturelle, Marginalisierung sind Facetten eines Phänomens, die sich wechselseitig verstärken 
können. Die Frage, ob und wie die digitale Spaltung zu schließen sei, kann nicht nur über den Einsatz von Technik beantwortet werden, sondern muss die anderen Facetten von Anfang an mit einbeziehen. Oft wird man bei Lösungsversuchen feststellen, dass die digitale Spaltung von den Betroffenen selbst nicht überwunden werden kann, sondern Hilfe von außen notwendig macht. Gleichzeitig aber darf daraus nicht der Schluss gezogen werden, dass es hinreichend sei, materielle Ressourcen von außen zu liefern - die Einbindung der Betroffenen und ihrer eigenen, oft nicht-materiellen Ressourcen, ist eine notwendige Bedingung für den Erfolg. Zusätzlich gilt, dass es keine „one size fits it all“-Lösung geben kann: Die Überwindung der digitalen Spaltung kann nur durch Berücksichtigung des jeweils gegebenen kulturellen Kontextes gelingen. Dies soll in den folgenden Abschnitten deutlicher gemacht werden.

\section{Sozioökonomische, politische und kulturelle Faktoren der digitalen Spaltung}

Es wäre verfehlt, die (globale) digitale Spaltung ausschließlich als ein Problem der fehlenden technischen Ressourcen zu sehen, doch unwichtig sind diese deshalb nicht. Praktisch die gesamte Informations- und Kommunikationsindustrie und der größte Teil der entsprechenden Infrastruktur und vor allem der Produkte und Anwendungen sind auf die Bedürfnisse und Anwendungskontexte der Nutzer in den entwickelten Ländern des Globus ausgerichtet: „[...] such technology is generated in and for the circumstances prevailing in the developed rather than the developing countries." (James 1999: 155). Dies macht sich an zunächst sehr trivialen Dingen fest: Ein Computer (inklusive der notwendigen Peripherie - Bildschirm, Tastatur, Maus, Drucker), der dem Stand der Technik entspricht und für den Endverbrauchermarkt zu günstigen Preisen hergestellt wird, kostet in der Bundesrepublik Deutschland zurzeit rund 600 bis 700 Euro. Vergleicht man dies bspw. mit dem durchschnittlichen Jahreseinkommen von 315 US-Dollar der Menschen in Afrika (Habitat 2001: 17), dann wird sehr schnell deutlich, dass dieser Preis die finanziellen Möglichkeiten vieler Menschen in den sich entwickelnden Ländern weit übersteigt. Ähnliche, wenngleich nicht ganz so dramatische Aussagen lassen sich für Teile der Bevölkerung von ökonomisch leistungsfähigen Industriestaaten wie den USA oder der Bundesrepublik Deutschland treffen, insbesondere aber auch für die Transformationsländer Mittel- und Osteuropas.

Doch nicht nur die Verfügbarkeit über die nötige Computertechnik als solche stellt eine große Hürde dar, sondern darüber hinaus auch die zum Betrieb notwendige Infrastruktur. ${ }^{2}$ Moderne Computer, gleich ob für den persönli-

2 Hier seien nur zwei Beispiele genannt, die die äußerst problematischen infrastrukturellen Bedingungen in vielen Entwicklungs- und Schwellenländern beleuchten sollen: So nennt Verzola (1999: 194) die Summe von 37 Mrd. US-Dollar, die nötig wären, um 51\% der phil- 
chen Gebrauch oder für den Einsatz in Industrie, Gewerbe, Handel oder Verwaltung, haben einen vergleichsweise großen Stromhunger - dies gilt letztlich auch für die meisten tragbaren Computer, die zudem in der Anschaffung deutlich teurer sind. In vielen Entwicklungsländern ist aber der Zugang zu Energie ein ähnlich großes Problem wie der Zugang zum Telefon. Selbst wenn Geräte vorhanden sind, können sie nicht ohne weiteres betrieben werden. Noch eine letzte Hürde: Marktübliche Geräte werden für Büros und Wohnungen in den gemäßigten Breiten entworfen, sie sind nicht für den Betrieb unter großer Feuchtigkeit oder Hitze oder für die Bedingungen häufiger Sandstürme etc. gebaut. Unter solchen Umweltbedingungen brechen handelsübliche Geräte schnell zusammen. Entsprechend angepasste Geräte wiederum sind weitaus teurer.

Neben den eher ökonomischen und infrastrukturellen Faktoren, die dazu führen, dass die digitale Spaltung existiert, können politische Ursachen identifiziert werden, die ebenfalls einen Beitrag zur Ungleichverteilung der Internetzugänge liefern. Viele Regierungen wollen aus politischen bzw. religiösen Gründen verhindern, dass die Bevölkerung der entsprechenden Länder Zugang zum Internet bekommen - bezüglich der politischen Motivation ist hier bspw. China zu nennen. ${ }^{3}$ Denn tatsächlich besitzt das Internet das Potenzial, Befreiungsbewegungen effektiv und effizient zu koordinieren, Informationsmaterial gegen totalitäre und diktatorische Regimes zu verbreiten oder Wahlkämpfe in Transformationsstaaten zu koordinieren - für letzteres ist Südkorea ein gutes Beispiel. Religiöse und politische Gründe sind hierbei in der Regel nicht klar zu trennen; meist sind Staaten, in denen eine fundamentalistische Religion vorherrscht, gleichzeitig nicht gerade unter die liberalen Staaten zu zählen. ${ }^{4}$

Fokussiert man nicht die ganze Welt, sondern beschränkt sich bei der Betrachtung auf einen Kontinent, so stößt man auf ähnliche Disparitäten. Global gesehen muss Europa als Wohlstandsregion bezeichnet werden, doch existiert dort zwischen und innerhalb von Ländern ebenfalls ein erhebliches

ippinischen Haushalte „mit den grundlegenden, aktuellen Informations- und Computertechnologien auszustatten" - sehr viel Geld für ein Land, in dem bereits die Mittel dafür fehlen, allein die Hauptstadt Manila ausreichend mit Trinkwasser akzeptabler Qualität zu versorgen. Die Situation auf dem afrikanischen Kontinent ist ähnlich desaströs, denn dort teilten sich 2002, ,[...] 750 Millionen Einwohner [...] nicht mehr als 20 Millionen Festnetz-Telefonanschlüsse - weniger als in Tokio. Und von diesen Telefonen steht der allergrösste Teil in den Hauptstädten. In den ländlichen Gebieten müssen sich im Durchschnitt mehr als 100 Leute einen Anschluss teilen - und der ist auch noch häufig gestört." (Asendorpf 2002).

3 Xiaoming und Kay (2004) widersprechen allerdings der Ansicht, dass die Schließung der digitalen Spaltung an Demokratisierung bzw. politische Freiheit von Staten gebunden sei; auch Analphabetismus und fehlende Englischkenntnisse sehen sie nicht als Hinderungsfaktoren für die Internetnutzung.

4 Bei diesen Bemerkungen wird die Standpunktabhängigkeit von Wertungen deutlich: Die teilweise virtuose Benutzung des Internet durch Islamisten und dem islamisch-fundamentalistischen Terror nahe stehenden Organisationen wird von den sie betreibenden Personenkreisen sicherlich als Werkzeug zur Befreiung angesehen; aus westlicher Perspektive hingegen stellen die entsprechenden Inhalte einen Aufruf zur menschenverachtenden Gewalt dar. 
Wohlstandsgefälle - sowohl entlang einer Nord-Süd- als auch einer Ost-WestAchse. Die Transformationsländer Mittel- und Osteuropas, die erst seit weniger als zwei Jahrzehnten marktwirtschaftlich organisiert sind und liberale Demokratien entwickeln, hinken immer noch deutlich hinterher, was den Zugang zum Internet betrifft (Ottens 2006). Die entsprechenden Zahlen spiegeln wieder, dass die ökonomische Situation in jenen Ländern bis heute alles andere als komfortabel ist; gleichzeitig wirken hier die überwundenen sozialistischen Regimes mit ihrer restriktiven Medienzugangspolitik nach - durch Seilschaften, die den politischen Wandel überlebt haben oder durch eine nun eher konservative oder nationalistisch ausgerichtete Medienkontrolle, wie sie zurzeit in Polen zu beobachten ist. Die Nord-Süd-Teilung Europas hinsichtlich der Internetzugänge ist zum Teil ebenfalls ökonomischen Ursachen geschuldet - allerdings liegen diese historisch weiter zurück: Industrialisierungsprozesse haben im Norden weit früher begonnen, Griechenland, Portugal, Spanien, Teile Italiens und Frankreichs waren bis weit in die zweite Hälfte des 20. Jahrhunderts agrarisch ausgerichtet. Zudem liegt die Vermutung nahe, dass kulturelle und sozialgeographische Faktoren eine Rolle spielen - die Aufrechterhaltung sozialer Beziehungen ist in Ländern wie Finnland, Schweden oder Norwegen mit einer geringen Bevölkerungsdichte und jahreszeitlich teilweise extremen Umweltbedingungen ohne die Nutzung moderner Kommunikationsmittel ohne Zweifel schwieriger als in Portugal oder Spanien. Gleichzeitig könnte es sein, dass Oralität und face-to-face-Kommunikation in Ländern Südeuropas eine größere Rolle als im Norden spielen.

Neben im weitesten Sinne ökonomischen und politischen Ursachen der digitalen Spaltung lassen sich zudem kulturelle Faktoren identifizieren. Demmers und O’Neil (2001) sprechen davon, dass im Internet zwei grundsätzlich verschiedene Nutzerkulturen aufeinander stoßen: „Takers“ auf der einen und „Leavers“ auf der anderen Seite (2001: 42f.). Diese der Ökologie entlehnten Ausdrücke bezeichnen den Umgang mit natürlichen Ressourcen: Takers sind Menschen bzw. deren Kulturen, die Ressourcen ausbeuten und verbrauchen sowie auf rasantes Bevölkerungs- und Wirtschaftswachstum ausgerichtet sind; Leavers hingegen entnehmen der Natur gerade nur soviel, wie sie für das tägliche Auskommen benötigen, und streben einen stabilen und ausgeglichenen Stoffkreislauf an, der die Umwelt weitgehend so belässt, wie sie ohne menschlichen Einfluss sein würde. ${ }^{5}$ Gerade in vielen Entwicklungsländern droht jenen, meist indigenen Völkern die kulturelle oder gar physische Auslöschung, weil ihr Territorium von Land suchenden Bauern oder Bodenschätze schürfenden

5 Diese Darstellung ist ohne Zweifel vereinfachend und es ist zu fragen, ob die beiden Autoren hier nicht einer bestimmten Perspektive auf so genannte „Naturvölker“ verpflichtet sind, die man durchaus als „romantisch“ bezeichnen könnte. Unbestritten bleibt aber, dass die Art und Weise der Produktion in den Industriestaaten vor allem in der Vergangenheit auf Ressourcenverbrauch und nicht auf deren Erhalt ausgerichtet ist. 
Unternehmen beansprucht wird. Sie verlieren ihre Existenzgrundlage, sind neuen Krankheiten ausgeliefert und können ihre tradierten Lebensformen nicht mehr verfolgen. Selbst wenn die betroffenen Menschen die Möglichkeiten hätten, bspw. über das Internet auf ihre Situation hinzuweisen, um so Aufmerksamkeit zu erregen, würde ihnen dies nur gelingen, wenn sie dies nicht in ihrer eigenen Sprache, sondern am besten in Englisch täten. Sprachbarrieren im Internet seien also wesentliche Ursachen für die digitale Spaltung nicht nur bezogen auf indigene Völker, sondern allgemein für alle Menschen, die keine englischen Muttersprachler sind (Demmers/O’Neil 2001: 56; widersprechend Xiaoming/Kay 2004). Eine zweite wichtige Ursache sehen Demmers/O’Neil (2001: 57) im tiefen Misstrauen vieler indigener Völker, das historisch durch Kolonialisierung, Ausbeutung oder gar Versklavung in der Vergangenheit entwickelt wurde. Technologie und somit auch das Internet würden, so eine These der Autoren, oft als weiteres Mittel der Ausbeutung vor allem des kulturell tradierten Wissens betrachtet und deshalb abgelehnt. Ergänzend bemerken sie (2001: 51ff.), dass die verfügbaren Benutzerschnittstellen von Computern den kulturellen und sozialen Traditionen vieler Menschen nicht angepasst seien. Die stark textorientierte Nutzung von Computern und dem Internet, so die These, würde der oralen Ausrichtung vieler Kulturen nicht gerecht werden. ${ }^{6}$

\section{Zwei Fallbeispiele}

Die digitale Spaltung muss nach dem bisher Gesagten als multifaktoriell verursachtes soziales Phänomen angesehen werden, das eben nicht nur auf fehlender oder mangelhafter Technologie beruht und daher über die Verfügbarkeit solcher Technologie lösbar wäre. Betrachtet man jedoch konkrete Projekte, die zur Schließung der digitalen Spaltung beitragen sollen, wird deutlich, dass hierbei oft, mehr implizit als explizit, jener oben skizzierte Technikdeterminismus vorausgesetzt wird bzw. die nicht-ökonomischen und nichttechnischen Ursachen der digitalen Spaltung unbeachtet bleiben. Dies soll nun an zwei Beispielen verdeutlicht werden: dem indischen Simputer- und dem derzeit verfolgten 100-Dollar-Laptop-Projekt.

\section{Der Simputer}

Der so genannte „Simputer“ (engl.: „Simple Computer“) ${ }^{7}$ wurde 1998 von einer Gruppe von IT-Fachleuten in Indien mit dem Zweck entwickelt, „to deve-

6 Es ist erstaunlich, dass das Thema der kulturell adäquaten Schnittstellengestaltung eher im Zusammenhang mit behinderten Personen diskutiert wird (z.B. Abascal/Nicolle 2005; Ford 1999). Dies könnte ein Hinweis darauf sein, dass die Perspektive auf solche Fragen und deren Bedeutung sehr stark durch die Herkunft der jeweiligen Autoren geprägt ist.

7 Der Simputer hat es sogar ins deutsche öffentlich-rechtliche Fernsehen geschafft: In einer 
lop a machine that is low-cost, usable and useful to the common man" (Manohar 1998: 1). Zielgruppe war insbesondere die ländliche Bevölkerung, die in aller Regel vom Zugang zu Computern und Informationen abgeschnitten ist. Ursache hierfür ist der Teufelskreis von Armut, geringem Bildungsgrad und Analphabetismus; zudem existiert in Indien als Entwicklungs- bzw. Schwellenland außerhalb der großen Ballungszentren kaum die für die Nutzung des Internet erforderliche Infrastruktur. Die Steuerung des Simputers sollte auf Piktogrammen aufbauen, um auf diese Weise Analphabeten die Nutzung ohne Hilfestellung zu ermöglichen. Zudem sollte das Gerät Texte in den in Indien verbreiteten Sprachen vorlesen können, um so Sprachbarrieren durch die Vielzahl der in Indien gesprochenen Idiome zu überwinden. ${ }^{8}$ Verschiedene Benutzerprofile sollten zu speichern sein, um den Austausch des Gerätes zwischen mehreren Nutzern zu ermöglichen. Ein Internetanschluss über Telefon sollte den Zugang zu zielgruppenspezifischen Informationsangeboten ermöglichen. Die Software sollte als Open Source entwickelt werden, um Lizenzgebühren zu sparen und zudem die Weiterentwicklung firmenunabhängig gestalten zu können, etwa durch NGOs oder Personen aus der Open Source- bzw. Free Software-Community. Um dem meist sehr niedrigen Einkommen der Zielgruppe des Simputers gerecht werden zu können, sollte das Gerät ca. 5.000 indische Rupien (am 15.08.2006 ca. 84 Euro, drei Jahre vorher ca. 96 Euro) kosten - allerdings widersprechen sich die Quellen in diesem Punkt: Priya Ganapati (2003) nennt in seinem Artikel 9.000 Rupien. Bei der Preisfindung wurde aber in jedem Fall von mindestens 100.000 verkauften Einheiten ausgegangen. Das Entwicklungsteam hatte sogar die Hoffnung, dass - sofern die angeführten technischen Spezifikationen erfüllt und der Preis gehalten werden könnte - es womöglich Hunderttausende oder gar Millionen Käufer geben würde. Da ein Preis von 5.000 Rupien als unteres Limit für die meisten indischen Bauern faktisch unerschwinglich und selbst für die untere Mittelschicht sehr teuer gewesen wäre, war zudem angedacht, dass Dorfgemeinschaften, Genossenschaften o. Ä. das Gerät kollektiv erwerben und benutzen sollten; eine andere Idee war, dass Kleingewerbetreibende das Gerät über ein Leihsystem einer größeren Zahl von Menschen zur Verfügung stellen. Benutzerprofile sollten dazu auf externen Speicherkarten abgelegt werden; die einzelnen Nutzer hätten somit nur vergleichsweise günstige Medien kaufen müssen, um ihre per-

Dokumentation über Indien (erneut gesendet von Phoenix am 5.10.2006) wurde über die Ziele und das Scheitern des Projekts berichtet, ebenso über das Nachfolgeprojekt „Mobilis“, das Ähnlichkeiten zum 100-Dollar-Laptop-Projekt aufweist (siehe http://www.ncoretech. com/mobilis/pdf/mobilisbrochure.pdf, zuletzt besucht am 5.10.2006).

8 Gemeint ist allerdings keine Übersetzungsfunktion (diese ist zurzeit angemessen kaum technisch realisierbar). Die Idee war vielmehr, dass in einer bestimmten Sprache verfasste Texte vorgelesen werden könnten: Der Simputer sollte also einen für verschiedene Sprachen geeigneten Sprachsynthesizer besitzen. So sollten wiederum Analphabeten in die Lage versetzt werden, für sie ansonsten nicht zugängliche Informationsangebote nutzen zu können. 
persönlichen Daten sichern zu können. Doch faktisch existiert der Simputer in der beschriebenen Fassung bis heute nicht. Zwar wurden von der Firma Encore etwa 1.500 bis 2.000 Geräte hergestellt - eine Quelle (Gongolsky 2004) spricht hier sogar nur von Vorbestellungen - und zu Preisen zwischen 12.000 (am 15.08.2006 ca. 202 Euro) für die einfachste und 22.000 indischen Rupien (am 15.08.2006 ca. 371 Euro) für die teuerste Ausstattung verkauft (Ganapati 2003), doch dies erreicht nicht einmal im Ansatz die erwarteten Verkaufszahlen und der Preis lag selbst für die einfachste Ausführung deutlich höher als ursprünglich geplant.

\section{Der 100-Dollar-Laptop}

Bevor auf die konzeptionellen Probleme der Projekte zur Schließung der digitalen Spaltung eingegangen werden kann, soll nun noch ein Projekt skizziert werden, das sich noch im Anfangsstadium befindet; daher ist es zu früh, von Erfolg oder Scheitern zu sprechen. Die Parallelen des Simputer-Projekts zum 100-Dollar-Laptop sind jedoch so augenscheinlich, dass die Prognose, dass der 100-Dollar-Laptop ebenfalls zum Scheitern verurteilt ist, durchaus nahe liegt. Das Projekt wird von Nicolas Negroponte vom MIT Media Lab vorangetrieben, geht aber auf Alan Kay zurück. ${ }^{9}$ Die Grundidee ist, dass möglichst allen Schülern in Entwicklungsländern ein Laptop für Bildungszwecke zur Verfügung gestellt werden soll, wobei die jeweiligen Regierungen die Geräte kaufen und dann an die Schüler verteilen sollen. Sein Design ist dabei so ausgelegt, dass der Laptop mit den oben angesprochenen schwierigen infrastrukturellen Bedingungen kompatibel ist. Er soll aus möglichst günstigen Komponenten gebaut werden, um den Preis niedrig halten zu können; dies soll auch dadurch unterstützt werden, dass Betriebssystem und Anwendungsprogramme Open Source sind. Nachdem das Unterfangen zunächst eher belächelt wurde, haben die PR-Aktivitäten Negropontes, insbesondere rund um den WSIS 2005, Wirkung gezeigt: Interviews und Berichte etwa in Wired (Poulsen 2005) konnten Öffentlichkeit und Aufmerksamkeit erzeugen. Nach den zahlreichen Meldungen, die derzeit (Anfang August 2006) im Internet kursieren, ist aber festzustellen, dass bisher keine Vereinbarungen mit Regierungen über Bestellungen oder gar Lieferungen getroffen wurden.

\section{Der Versuch einer Evaluation}

Betrachtet man die beiden skizzierten Projekte und beachtet dabei die oben angeführten Ursachen für die digitale Spaltung, so ist das Scheitern des Simputer-Projekts in der Retrospektive kaum mehr überraschend; ebenso nahe lie-

9 Die englische Bezeichnung des Projekts lautet „One Laptop per Child“ und geht damit nicht so sehr auf den Preis des Geräts sondern auf Adressaten des Projekts ein. Siehe hierzu und zum Folgenden OLPC (2006), insbesondere die Wiki-Einträge. 
gend ist Skepsis gegenüber dem 100-Dollar-Laptop. So haben beide Entwürfe gemeinsam, dass die geplanten Geräte letztlich nicht dem Stand der Technik entsprachen bzw. entsprechen. Der Simputer sollte zu Beginn ein technisch sehr einfaches Gerät sein - erst spätere Entwürfe wurden komplexer. Der 100Dollar-Laptop wiederum benutzt ganz augenscheinlich Hardware, die in Industrieländern vor vier bis fünf Jahren dem Stand der Technik und den entsprechenden Nutzeranforderungen entsprachen, aber heute zum alten Eisen gehören. Für den psychologischen Effekt dieser Design-Entscheidung gibt es zwar keine Belege, aber es ist durchaus zu vermuten, dass viele potentielle Anwender dies als Herabsetzung empfinden; es könnte der Eindruck entstehen, dass das Projekt nur dazu dient, für in Industrieländern ansonsten unverkäufliche Produkte im großen Maßstab Abnehmer zu finden. Allerdings ist diese Einschätzung spekulativ.

Obwohl der Simputer in Indien selbst entwickelt wurde, krankt dieses wie das Laptop-Projekt daran, dass die potenziellen Nutzer nicht an der Entwicklung der Geräte beteiligt waren oder sind. Das kann recht gut an den jeweiligen Designs zur Vernetzung der Geräte gesehen werden. Der Simputer sollte per Telefon Zugang zum Internet und seinen Informationsangeboten finden; diese gehörten zum Kern des Projekts, denn den Nutzern sollten auf diese Weise aktuelle Informationen zu Preisen ihrer eigenen Agrarprodukte, zum Wetter, Tipps zur Ernte etc. bereitgestellt werden. Ohne den entsprechenden Informationszugriff war der Nutzen des Geräts eher gering bzw. kaum mehr gegeben. Doch gerade der Telefonzugang ist in Indien auf dem Land schwierig. Der 100Dollar-Laptop wiederum soll per WLAN vernetzt werden. Bedenkt man jedoch, dass die Reichweite hierbei eher im Meter- denn im Kilometerbereich liegt, bedeutet dies, dass zwar einige Geräte untereinander in Kontakt treten könnten, aber oft kein Zugriff zum Internet hergestellt werden kann - mit einer geographischen Metapher gesprochen: Die so entstehenden Netze stellen Inseln ohne jede Verbindung zum Festland dar, denn in vielen Teilen der Welt leben in Bezug auf Telekommunikation viele Menschen sehr abgeschieden. Es müssten also zunächst erhebliche infrastrukturelle Maßnahmen ergriffen werden, um die geplanten Nutzungsszenarien überhaupt möglich zu machen. Dies jedoch relativiert den angepeilten Preis gerade des 100-Dollar-Laptop, da die Kosten jener Infrastruktur anteilig auf jedes Gerät umgelegt werden müssten. Nun laufen diese Bemerkungen bisher auf das Plädoyer hinaus, dass in den betreffenden Projekten die Technik nur falsch konzipiert wurde - wären die Designentscheidungen richtig getroffen worden, könnten solche Projekte erfolgreich sein. Das wäre jedoch zu kurz gegriffen und ginge an den Problemen vorbei. Denn bei der digitalen Spaltung handelt es sich nicht nur um ein Problem der fehlenden Wirtschaftskraft oder technischen Infrastruktur. Deutlicher: Dies sind zwar wichtige, aber doch nachgeordnete Faktoren. In Technology and Social Inclusion demonstriert Mark Warschauer (2003: 1ff.) dies an 
ganz unterschiedlich gelagerten Beispielen, wobei hier stellvertretend auf eines davon eingegangen werden soll (Warschauer 2003: 2). Er beschreibt das Projekt eines Computerkiosks, das in einem Slum von Neu Delhi durchgeführt wurde. Die Grundidee dabei war, ökonomisch und vor allem sozial marginalisierten Menschen Zugang zu Computern und Internet anzubieten. Dazu wurde ein Kiosk eingerichtet, tatsächlich war dies im Grunde nur ein Loch in einer Wand, und den Nutzern Zugriff auf einen Bildschirm und einen speziellen Joystick als Eingabeschnittstelle gewährt; Maus oder Tastatur gab es nicht. Ebenso wurden keinerlei Schulungen oder Unterweisungen der Nutzer angeboten; der Internetzugang funktionierte aufgrund technischer Probleme selten. Ergebnis war, dass „, [c]hildren did learn to manipulate the joysticks and buttons, but almost all their time was spent drawing with paint programs or playing computer games." Warschauer berichtet darüber, dass viele Eltern Bedenken äußerten, da sie keinen Nutzen in dem Projekt sehen konnten oder darin sogar einen Schaden erkannten, da sich die Schulleistungen ihrer Kinder aufgrund der Vernachlässigung der Hausarbeiten zugunsten des Zeitvertreibs am Computerkiosk verschlechterten. ${ }^{10}$

Dieses Beispiel stellt ein starkes Indiz dafür dar, dass die Einbindung der jeweiligen Zielgruppe und die Entwicklung eines pädagogischen Konzepts zentrale Vorbedingungen für den Erfolg entsprechender Projekte zur Schließung der digitalen Spaltung darstellen. Bezüglich des Simputers und des 100-DollarLaptops ist zu vermuten, dass die Adressaten darauf abheben würden, dass nicht der Besitz eines Computers oder Laptops ihr zentrales Anliegen sei, sondern der Zugang zu Bildung - und vielleicht auch noch: der Zugang zu sauberem Wasser, ausreichender Nahrung, angemessener Gesundheitsversorgung, politischer Partizipation, rechtsstaatlicher Behandlung, kultureller Autonomie, Frieden und Sicherheit, Zugang zu Märkten für ihre eigenen Produkte - die Liste, die von den Betroffenen aufgestellt werden würde, wäre sicherlich noch umfassender. An Entwicklungshilfeprojekten zur Vergabe von Mikrokrediten wird gut ersichtlich, dass nicht technische Geräte, sondern vor allem die Veränderung sozioökonomischer Bedingungen einen wesentlichen Beitrag zur Verbesserung der Lebensbedingungen liefern können (vgl. UNCDF 2006). Verfolgt man rückblickend die Entwicklung des Simputers und aktuell jene des 100-Dollar-Laptops, so ist erkennbar, dass beide Projekte letztlich auf der Sichtweise aufbauen, dass es ausreiche, Menschen Technik zur Verfügung zu stellen, um ihre im weitesten Sinne sozialen Probleme zu lösen. Entscheidend für den Erfolg von Projekten zur Schließung der digitalen Spaltung ist für

10 In der schon genannten Fernsehsendung wurde auch über dieses Projekt (http://www.holein-the-wall.com/ zuletzt besucht am 05.10.2006) berichtet. Die dort gezeigten Bilder stehen im Widerspruch zu Warschauers Beschreibung, so besitzen die Computer z.B. Tastaturen. Die Projektmitarbeiter bewerten, vielleicht wenig überraschend, das Projekt rundweg positiv - und sind ganz offensichtlich einer technikdeterministischen Perspektive verpflichtet. 
Warschauer (2003: 199ff.) jedoch die Einsicht, dass Technik sozial eingebettet sein muss. Der Versuch, Technologie einfach zur Verfügung zu stellen und zu warten, dass sich zu dieser „Lösung“ dann schon die passenden Problemstellungen finden werden, muss - dies wurde an den weiter oben aufgeführten Beispielen bereits deutlich - scheitern. Die beiden konträren Auffassungen werden in der folgenden Gegenüberstellung deutlich (in Anlehnung an Warschauer 2003: 207).

\begin{tabular}{|l|l|}
\hline Standard-Werkzeug-Modell & Soziotechnisches Modell \\
\hline IuK-Technologie ist ein Werkzeug. & $\begin{array}{l}\text { IuK-Technologie stellt ein soziotechnisches } \\
\text { Netzwerk dar. }\end{array}$ \\
\hline Ein Geschäftsmodell ist ausreichend. & $\begin{array}{l}\text { Die Beachtung von Umwelt- und Umgebungs- } \\
\text { bedingungen ist notwendig. }\end{array}$ \\
\hline $\begin{array}{l}\text { Es werden einmalige Installationen von } \\
\text { IuK-Technologie durchgeführt. }\end{array}$ & $\begin{array}{l}\text { Der Einsatz von IuK-Technologie stellt einen } \\
\text { sozialen Prozess dar. }\end{array}$ \\
\hline Technik wirkt direkt und sofort. & $\begin{array}{l}\text { Technik wirkt indirekt und auf verschiedenen } \\
\text { Zeitskalen. }\end{array}$ \\
\hline $\begin{array}{l}\text { Politische Faktoren sind schlecht oder } \\
\text { zumindest irrelevant. }\end{array}$ & $\begin{array}{l}\text { Politische Faktoren sind zentral und ermögli- } \\
\text { chen oft überhaupt erst einen Erfolg. }\end{array}$ \\
\hline $\begin{array}{l}\text { Anreize zum Wandel bringen keine } \\
\text { Probleme mit sich. }\end{array}$ & $\begin{array}{l}\text { Anreize benötigen möglicherweise weit reichen- } \\
\text { de Umstrukturierungen und können Konflikte } \\
\text { auslösen. }\end{array}$ \\
\hline $\begin{array}{l}\text { Beziehungen können leicht einseitig ver- } \\
\text { ändert werden. }\end{array}$ & $\begin{array}{l}\text { Beziehungen sind komplex, entstehen durch } \\
\text { Verhandlungen und beruhen auf verschiedenen } \\
\text { sozialen Mechanismen, bspw. Vertrauen. }\end{array}$ \\
\hline $\begin{array}{l}\text { Soziale Effekte der IuK-Technologie sind } \\
\text { zwar groß, aber isoliert und harmlos. }\end{array}$ & $\begin{array}{l}\text { IuK-Technologie kann enorme soziale Auswir- } \\
\text { kungen zeigen, die sich über alle Lebensberei- } \\
\text { che erstrecken können. }\end{array}$ \\
\hline $\begin{array}{l}\text { Die Einsatzbedingungen sind einfach } \\
\text { strukturiert (bspw. demografische Varia- } \\
\text { blen). }\end{array}$ & $\begin{array}{l}\text { Die Einsatzbedingungen sind komplex und um- } \\
\text { fassen bspw. Gefüge aus Unternehmungen, } \\
\text { Dienstleistungen, Menschen, Technik, Ge- } \\
\text { schichte und Orte. }\end{array}$ \\
\hline $\begin{array}{l}\text { Wissen und Know-how können leicht } \\
\text { expliziert werden. } \\
\text { IuK-Technologie allein reicht aus. } \\
\text { verborgen. Know-how sind meist implizit und }\end{array}$ \\
$\begin{array}{l}\text { IuK-Technologie wird nur in Kombination mit } \\
\text { anderen Fähigkeiten und Leistungen erfolgreich } \\
\text { eingesetzt werden können. }\end{array}$ \\
\hline
\end{tabular}

Wichtig an dieser Gegenüberstellung ist, dass die Maßnahmen zur Schließung der digitalen Spaltung die Intentionen der Adressaten in Rechnung stellen müssen. Es ist notwendig, diese in die Hilfsmaßnahmen zu involvieren, um jede Form des Paternalismus zu vermeiden, auch wenn diese Vorgehensweise meist sehr zeitaufwändig ist (Warschauer 2003: 200f.). Ebenso wichtig ist, die vorhandenen Fähigkeiten aller involvierten Menschen zu nutzen (vgl. Mansell/Steinmueller 2002: 37ff.): 
„A common mistake made in ICT development projects [IuK Entwicklungshilfeprojekten] is to make primary use of computer experts rather than of the best community leaders, educators, managers, and organizers. Those who are capable of managing complex social projects to foster innovative, creative, and social transformation will likely be able to learn to integrate technology into this task. [...] [L]ocal initiative is critical to give the space for good small things to emerge." (Warschauer 2003: 212)

Die Möglichkeit erfolgreicher Hilfe beruht damit auf Anerkennung, auf Reziprozität und Gleichheit. Maßnahmen zur Behebung sozialer Ungleichheiten wie der digitalen Spaltung müssen auf Empowerment zielen und dürfen nicht auf einer paternalistischen Attitüde beruhen. Ebenso muss beachtet werden, dass Technik und ihre Sinngebung, Deutung, Zwecke und Einsatzmöglichkeiten nicht einfach feststehen, sondern auf Akten der sozial bestimmten Interpretation und Konstruktion beruhen. ${ }^{11}$ Selbst wenn man nicht von einem erkenntnistheoretischen und moralischen Relativismus ausgehen will, darf nicht übersehen werden, dass viele Aspekte der Welt nicht einfach objektiv gegeben sind, sondern sozial konstruiert werden.

Hiermit ist allerdings auch ein erhebliches Problem verbunden. Soziale Defizite, die mit entsprechenden Projekten behoben werden sollen, beruhen nicht selten auf einem Machtgefälle (vgl. Moss 2002) zwischen Bevölkerungsgruppen oder auf Diskriminierungen etwa aufgrund des Geschlechts oder der sozialen Herkunft, in Indien insbesondere aufgrund der Kastenzugehörigkeit. Die Anerkennung bestehender sozialer Bedingungen würde jedoch bedeuten, im Rahmen dieser Diskriminierungen agieren zu müssen - etwa nur Männern den Zugang zu Computern und dem Internet zu verschaffen. Dies widerspräche aber der Idee des Empowerment marginalisierter Menschen. Das heißt: Jedes Entwicklungshilfeprojekt, nicht nur jene zur Schließung der digitalen Spaltung, bewegt sich hier auf einem schmalen Grat.

\section{Fazit}

Die digitale Spaltung ist eingebettet in eine Vielzahl von Faktoren und sozialen Bedingungen. Sie ist Ergebnis und Ausdruck vieler anderer Spaltungen, vertieft diese aber zusätzlich. Sie muss als Teil der viel weitergehenden Spaltung der Welt bezüglich des Zugangs zu Bildung und Wissen verstanden werden. Armut und Analphabetismus gehen nicht nur in den Ländern der so genannten „Dritten Welt“ Hand in Hand, sondern treten ebenfalls in Industrieländern oft gemeinsam auf. Mangelhafte Nahrungs- und Gesundheitsversorgung tragen ebenfalls dazu bei, dass die kognitiven Fähigkeiten der betroffenen Menschen verringert oder erst gar nicht entwickelt werden. Armut, Analphabetismus, Krankheit und Hunger verstärken sich zudem wechselseitig. Die

11 Solche Prozesse werden z.B. in der Actor-Network-Theory (ANT) und der Theorie der „Social Construction of Technology" (SCOT) beschrieben und konzeptionell erfasst (siehe hierzu Bijker/Law 1994; Oudshoorn/ Pinch 2003; Schulz-Schaeffer 2000). 
fehlende oder mangelhafte Infrastruktur für Transport, Verkehr und Kommunikation, oft verursacht durch widrige Umweltbedingungen und -katastrophen sowie nicht selten durch (Bürger-)Kriege, muss als weitere Ursache der sozialen Marginalisierung vieler Menschen in Entwicklungs- und Schwellenländern angesehen werden. Ohne stabile politische Verhältnisse in den betroffenen Ländern wird aber gerade diese Problematik nicht zu lösen sein. Durch die hohen Marktzugangsbarrieren, die die entwickelten Länder den Entwicklungs- und Schwellenländern in den Weg gestellt haben, sind diese außerdem nicht in der Lage, aus eigener Kraft fehlende Ressourcen zu erwirtschaften, um die zu geringen Ausgaben in Bereichen wie Bildung, Infrastruktur oder medizinische Grundversorgung selbst zu erhöhen. Restriktiver Zugang zu Informationen in Ländern mit politisch-autoritären und/oder religiös-fundamentalistischen Regimen verhindert selbst in weit vorangeschrittenen Schwellenländern wie China, dass sowohl Privatpersonen als auch Unternehmen alle Vorteile des gigantischen Informationspotenzials des Internets nutzen können.

Kurz: So lange medienwirksame Projekte wie der 100-Dollar-Laptop unter dem Vorbehalt stehen, dass sie Vorteile für die eigene Industrie, die eigene (Forschungs-)Institution o. Ä. bringen sollen und zudem von einer allzu enggefassten Vorstellung der rein technischen Behebung der digitalen Spaltung ausgehen, werden sie notwendigerweise scheitern oder zumindest für die anvisierten Adressaten keinen Nutzen bringen. Auch das Ziel des Empowerment stellt nicht mehr als hohle Rhetorik dar, so lange Hilfe paternalistisch-benevolent gewährt wird. Der Zugang zu moderner Technik kann (und muss) ein Mittel zur Verbesserung von Lebensbedingungen in weniger entwickelten Ländern sein, dies wird jedoch nur dann realisierbar, wenn von den konkreten sozialen, wirtschaftlichen und ökologischen Strukturen ausgegangen wird, auf die mit Hilfe der Technik bewahrend oder verändernd Einfluss genommen werden soll - und zwar mit Beteiligung der Betroffenen. Die Hoffnung, Technik könne soziale Probleme gleichsam im Alleingang lösen, bleibt eine Illusion.

\section{Literatur}

Abascal, J.; Nicolle, C. (2005): Moving towards inclusive design guidelines for socially and ethically aware HCI, in: Interacting with Computers, Vol. 17, 484-505.

Asendorpf, D. (2002): Langsam, teuer, aber faszinierend. Auch in Afrika setzt sich das Internet zunehmend durch, in: Neue Zürcher Zeitung (NZZ), 20.09.2002.

Bijker, W.; Law, J. (1994, eds.): Shaping Technology, Building Society. Cambridge/Massachusetts, London/England.

Carlisle, Y. M.; Manning, D. J. (1999): Ideological persuasion and technological determinism, in: Technology in Society, Vol. 21, No. 1, 81-102.

Carson, R. (1962): Silent spring. Boston.

Castells, M. (2001): The Internet Galaxy. Oxford.

Darkwa, O. K.; Eskow, St. (2000): Creating an African Virtual Community College: Issues and Challenges, in: First Monday, Vol. 5, No. 11, http://www.firstmonday.org/issues /issue5_11/ darkwa/ zuletzt besucht am 2.7.2006.

Darkwa, O. K.; Mazibuko, F. (2000): Creating Virtual Learning Communities in Africa: Chal- 
lenges and Prospects, in: First Monday, Vol. 5, No. 5, http://www.firstmonday.org/issues/ issue5_5/darkwa/, zuletzt besucht am 2.7.2006.

Demmers, J.; O’Neil, D. (2001): Leavers and Takers: Alternative Perspectives on Universal Access to Telecommunications Technologies, in: Techné, Vol. 5, No. 3, http://scholar.lib.vt.edu/ ejournals/SPT/v5n3/pdf/demmers.pdf zuletzt besucht am 13.8.2006.

Ford, P. (1999): Information Technologies, the Physically Disabled, and New Valuations, in: Pourciau, L. J. (ed.): Ethics and Electronic Information in the Twenty-First Century. West Lafayette/Indiana, 46-61.

Ganapati, P. (2003): Simputer: Not for the common man anymore, 17.9.2003, http://in.rediff.com/money/2003/sep/17spec.htm, zuletzt besucht am 14.8.2006.

Gongolsky, M. (2004): Der Simputer ist ausgeträumt, in: Netzkritik.de, http://www.netzkritik.de/ art/172.shtml zuletzt besucht am 13.8.2006.

Gurstein, M. (2003): Effective use: A community informatics strategy beyond the digital divide, in: First Monday, Vol. 8, No. 12, http://www.firstmonday.org/issues/issue8_12/gurstein/ zuletzt besucht am 2.7.2006.

Habitat (2001): Cities in a Globalizing World. Global Report on Human Settlements 2001. London, Sterling/Virginia.

James, J. (1999): Globalization, Information Technology and Development. London.

Manohar, S. (1998): The Simputer: access device for the masses, http://www.simputer.org/ simputer/history/paper.pdf zuletzt besucht am 14.8.2006.

Mansell, R.; Steinmueller, W. E. (2002): Mobilizing the Information Society. Oxford, New York.

Meadows, D.; Meadows, D.; Zahn, E.; Milling, P. (1972): The Limits to Growth. New York.

Moss, J. (2002): Power and the digital divide, in: Ethics and Information Technology, Vol. 4, No. 2, 159-165.

OLPC (2006): One Laptop per Child. http://www.laptop.org/ zuletzt besucht am 14.8.2006.

Ottens, M. (2006): Internetnutzung durch Privatpersonen und Unternehmen, in: Statistik kurz gefasst, 12/2006, http://epp.eurostat.cec.eu.int/cache/ITY_OFFPUB/KS-NP-06-012/DE/KSNP-06-012-DE.PDF zuletzt besucht am 14.8.2006.

Oudshoorn, N.; Pinch, T. (2003, eds.): How Users Matter. The Co-Construction of Users and Technologies. Cambridge, London.

Poulsen, K. (2005): Negroponte: Laptop for Every Kid, in: Wired, 17.11.2005, http://www.wired.com/news/technology/0,69615-0.html zuletzt besucht am 14.8.2006.

RIAP (2002): Alleviating the Digital Divide: Policy Recommendations. Research Institute for Asia and the Pacific, University of Sydney, http://www.riap.usyd.edu.au/research/bica/documents/ FinalReport.pdf zuletzt besucht am 2.7.2006.

Schulz-Schaeffer, I. (2000): Sozialtheorie der Technik. Frankfurt/Main, New York.

UNCDF (2006): International Year of Microcredit 2005 - Final Report. United Nations Capital Development Fund. New York.

USDC (2000): Americans in the information age: Falling through the Net. U. S. Department of Commerce, http://search.ntia.doc.gov/pdf/fttn00.pdf zuletzt besucht am 2.7.2006.

Verzola, R. (1999): Die Dritte Welt und das Internet: Das Beispiel Philippinen, in: Rötzer, F. (Hrsg.): Megamaschine Wissen. Frankfurt/Main, New York.

Warschauer, Mark (2003): Technology and Social Inclusion. Rethinking the Digital Divide. Cambridge/Massachusetts, London.

WSIS (2003): Declaration of Principles - Building the Information Society: a global challenge in the new Millennium. International Telecommunication Union, http://www.itu.int/dms_pub/ itu-s/md/03/wsis/doc/S03-WSIS-DOC-0004!!PDF-E.pdf zuletzt besucht am 2.7.2006.

Xiaoming, H.; Kay, Ch. S. (2004): Factors affecting Internet development: An Asian survey, in: First Monday, Vol. 9, No. 2, http://firstmonday.org/issues/issue9_2/hao/ zuletzt besucht am 15.8.2006.

Zachary, G. P. (2004): Black star: Ghana, information technology and development in Africa, in: First Monday, Vol. 9, No. 3, http://www.firstmonday.org/issues/issue9_3/zachary/zuletzt besucht am 2.7.2006. 